\title{
La propiedad Intelectual y su importancia en la Universidad Rodulio Perdomo
}

CIENCIA Y TECNOLOGÍA (CT) Entrevista al economista Rodulio Perdomo quien realizó estudios de Licenciatura en Economía y Maestría en Economía y Planificación del Desarrollo. Previo a la formación de la maestría desempeñó trabajos en la Secretaría de Finanzas como analista presupuestario y analista de ingresos fiscales.

En 1984 se trasladó a Francia, becado por el gobierno de ese país, y tras dos años de estudios obtiene el D.E.A. en Sistemas y Estructuras por la Universidad de París; correspondiente al primer nivel de estudios doctorales.

En Julio de 1986 se reincorporó a la Secretaría de Finanzas como Asesor de la Dirección General de Presupuestos. Actualmente labora en forma ininterrumpida como Docente Titular II. Realizó Talleres-Pasantías de dos meses en El Servicio de Impuestos Internos de la República de Chile, Pasantía (15 días) en la Secretaría de Hacienda Costa Rica, Curso de 1 mes: Economía Internacional de la Salud en la Universidad Central de Ecuador, Curso de Economía y Desarrollo de Sistemas Sanitarios (15 días) en la Universidad Estatal de Bahía, Brasil.

Como docente se ha desempeñado en el ámbito local e internacional. Actualmente se desempeña como docente de Economía Internacional, Estructuras de la Economía Internacional y de Honduras, coordinador del Proceso de Autoevaluación de la Carrera de Comercio Internacional de la Facultad de Economía UNAH.

CT/ ¿Qué importancia tiene la Propiedad Intelectual en un país como Honduras?

RP/ La Propiedad Intelectual es fundamental para generar y orientar el desarrollo de los países. Representa una porción de riqueza que puede o no develarse para sumarla al stock de riqueza producida por los países.

\section{CT/ ¿Es la Propiedad Intelectual un factor de desarrollo?}

RPI Todos los países que han alcanzado altos niveles de desarrollo tecnológico hacen de la PI un factor estratégico de desarrollo. Edison, a finales del siglo XIX, es un buen ejemplo de cómo hacer de la PI el núcleo de una riqueza que se sustenta en la creatividad humana para resolver problemas de la humanidad. 
CT/ ¿La Propiedad Intelectual es simplemente la protección de intereses patrimoniales de los países desarrollados?

RP/ La Propiedad Intelectual es, sobre todo, asegurar bases firmes de crecimiento y desarrollo. No tiene solamente una finalidad protectiva o de garantizar la exclusividad en el uso de una invención y la renta monetaria por dejar que otros usen esta invención pues aspira, sobre todo, a ampliar de manera exponencial la base de oportunidades para el desarrollo económico, social y tecnológico de los pueblos.

\section{CT/ ¿Tiene Honduras oportunidades reales de beneficiarse de la Propiedad Intelectual?}

RPI Sí tiene, partiendo primero de la organización de una plataforma de aprovechamiento integral de la Propiedad Intelectual. Esta plataforma representa la gestión ordenada del conocimiento tecnológico de un país para que, en adelante, los avances científicos tecnológicos del país se conviertan en una base mayor para los fines de agregación de valor a los bienes y servicios producidos o por producir.

\section{CT/ ¿Cómo puede Honduras crear condiciones que favorezcan la generación de activos intangibles, susceptibles de protección y transferencia?}

RPI El desarrollo de activos intangibles; pensando en inventos e innovaciones tecnológicas, parte primero de identificar aquello susceptible de incorporar en procesos de Propiedad Intelectual. Desarrollar un catálogo inicial de oportunidades por país, regiones, localidades y sitios específicos naturalmente tienen lugar estos procesos. (Institutos técnicos, laboratorios, fábricas, Cátedras universitarias, etc.).

\section{CT/ ¿Qué papel juega la Universidad en el desarrollo de activos intangibles?}

RPI La UNAH puede establecer una Estrategia de Largo Plazo para: identificar, desarrollar, promover y facilitar procesos de Propiedad Intelectual. Ello implica una unidad de técnicos bien capacitados para abrirse a las demandas de la población en general y en particular de los que ya producen y usan innovaciones científicotecnológicas. (Un profesor universitario genera vacunas a partir de procesos innovadores, otro ha encontrado materiales muy baratos para construir viviendas para los pobres, etc.).

CT/ ¿Cómo podría la Universidad crear liderazgo en la generación, protección, aprovechamiento y transferencia de conocimiento y tecnología?

RP/ Para crear liderazgo, primero hay que aparecer y demostrar liderazgo mediante la 


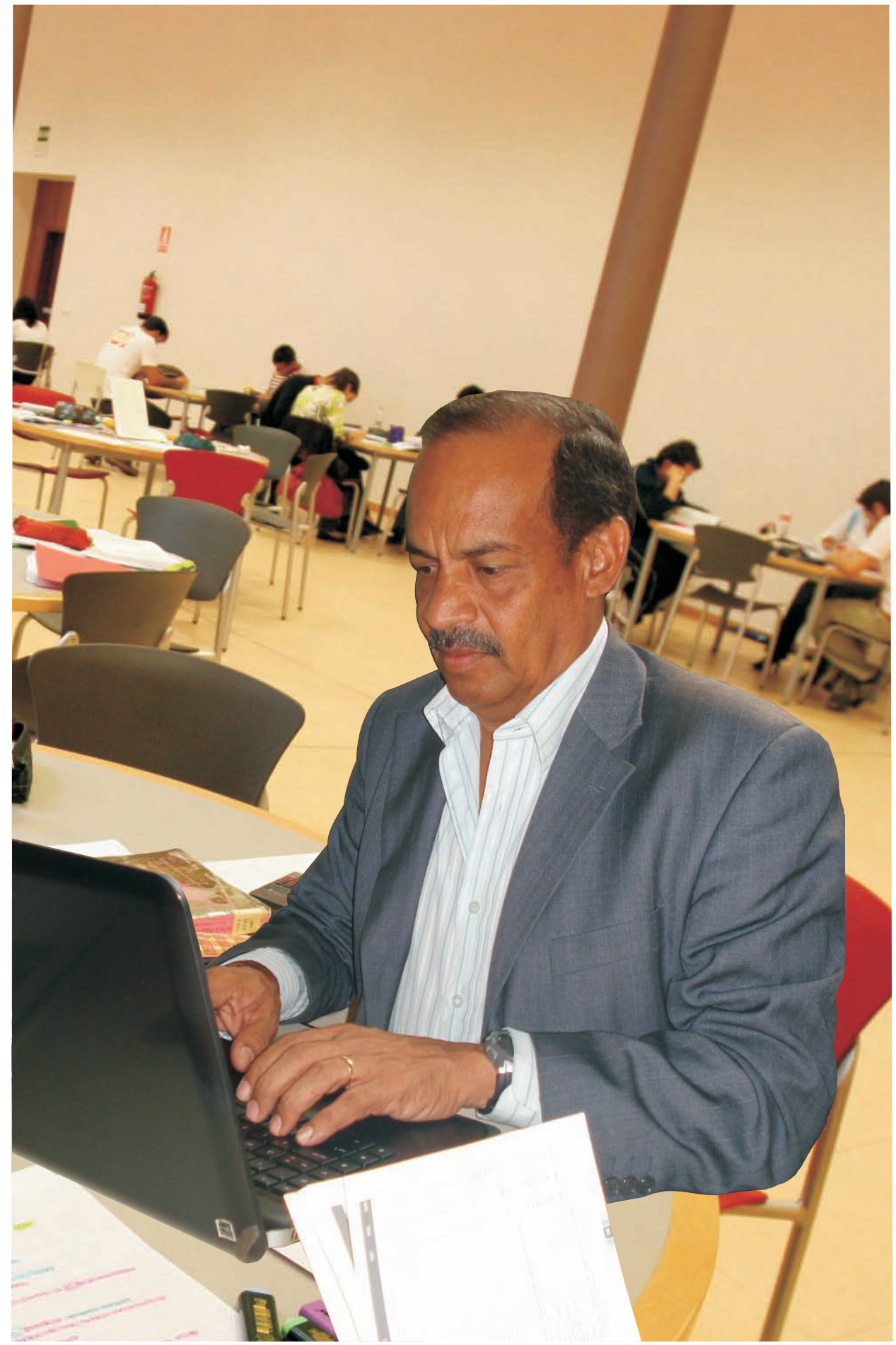

Dirección de Investigación Científica $\longdiv { 1 7 }$ 
apropiación de todo el espectro de actividades y procesos que intervienen en Propiedad Intelectual (expertos en marco legal nacional e internacional de la Propiedad Intelectual, investigadores de Propiedad Intelectual, equipo y software con servidores para crear base local de consultas, expertos en marketing y transacciones de Propiedad Intelectual, etc.)

\section{CT/ ¿Existen condiciones tecno-científicas y legales para la creación y desarrollo de empresas de base tecnológica en la UNAH?}

RP/ Existen parcialmente, aunque el resto puede crearse o suscitarse primero a partir de un marco legal que asegure a la UNAH disponer de una plataforma de operatividad que permita desplegar las potencialidades básicas necesarias.

\section{CT/ ¿Cuáles son los principales obstáculos que tiene la Universidad para el desarrollo tecnológico, la innovación y la Propiedad Intelectual?}

RPI No existen recursos presupuestarios en la UNAH para Propiedad Intelectual, tampoco personal que pueda dedicarse con exclusividad a estos menesteres porque el éxito radica en que exista una masa crítica dedicada por entero a tales actividades. Sin embargo, a partir de experiencias exitosas como el Consultorio Jurídico de la UNAH puede crearse un núcleo básico de personal especializado en dichas temáticas. Principal obstáculo: la concepción filosófica y política de la UNAH impide, para el caso, que se pueda establecer un convenio de mediano plazo con un grupo de empresas porque las empresas son capitalistas y el capitalismo es perverso 0 va enriquecer más a tal persona. El cambio de la concepción filosófica y política pasa por desterrar algunos fantasmas que animan las actitudes de muchos profesores universitarios (hay que darle vacaciones por un tiempo a: Bakunin, Lenin, Tchayanov, R. Luxemburgo, Trosky) y lograr que las nuevas actitudes propicien un desarrollo científico y tecnológico real y puesto al servicio de todos, aunque hayan opciones preferentes por los más pobres.

\section{CT/ ¿Qué recomendaría Usted, para que la Universidad tenga una Visión hacia la Sociedad del Conocimiento; comprometida con la generación, uso, aplicación y explotación del conocimiento?}

RPI Primero identificar unos 20 profesionales universitarios bien dispuestos, con nuevas actitudes y enfocados en avanzar cada vez más en la temática de la Propiedad Intelectual. Ya la Dirección de Investiagación Científica arrancó con estos procesos, sólo les queda dar los pasos subsiguientes. 